\title{
EFFECTS OF SOME ANTIDIABETIC AND ANTIHYPERTENSIVE DRUG COMBINATIONS ON STREPTOZOTOCIN-INDUCED DIABETIC RATS
}

\author{
Olapeju I. Bolanle, Eric K. I. Omogbai, Enitome E. Bafor \\ Pharmacology \& Toxicology, University of Benin, Nigeria
}

Background: Diabetes mellitus is a spectrum of common metabolic disorders, arising from a variety of pathologic mechanisms, all resulting in hyperglycaemia. Diabetes and hypertension frequently occur together and are two leading risk factors for atherosclerosis and its complications. This study examined the effects of some antidiabetic (metformin and glibenclamide) and antihypertensive (amlodipine and valsartan) combination regimens on streptozotocin-induced diabetes mellitus in rats.

Method: Male Wistar rats weighing (200 g to $350 \mathrm{~g}$ ) were fasted overnight and diabetes mellitus was induced by a single dose $(40 \mathrm{mg} / \mathrm{kg}$ body weight) intraperitoneal injection of streptozotocin dissolved in a freshly prepared $0.1 \mathrm{M}$ citrate buffer, pH 4.5. After $48 \mathrm{~h}$, diabetes mellitus was confirmed (blood sugar \&\#8805; $200 \mathrm{mg} / \mathrm{dl}$ ) and the animals were grouped into eight groups of seven rats each that included non-diabetic control rats with no drug treatment, untreated diabetic animals and other groups which were treated with different combination regimens. After six weeks treatment, animals were sacrificed under chloroform anaesthesia, visceral organs (which included kidney, liver, lung and heart) and blood samples were collected for histology and biochemical analyses respectively. Statistical analysis was done using oneway analysis of variance followed by Dunnett's post-hoc test of multiple comparison (Graphpad Prism\&\#174; 6 San Diego, USA). P-value was set at $\mathrm{P}<0.05$ for significant difference.

Results: Diabetic rats had 100\% mortality but addition of antihypertensives (amlodipine or valsartan) to antidiabetics (metformin or glibenclamide) improved survival rate as seen in glibenclamide plus valsartan treated group which had $71.4 \%$ compared with $57.4 \%$ in glibenclamide-treated. The addition of the antihypertensives significantly $(\mathrm{P}<0.05)$ improved blood glucose control (except glibenclamide+amlodipine treated)

and ameliorated pathologic changes in the biochemical indices, haematological counts, liver function, renal function and lipid profile, $(\mathrm{P}<0.05)$. There was also histologic evidence of organ protection among the various treated groups when compared with the untreated diabetic group.

Conclusion: Addition of amlodipine or valsartan to metformin or glibenclamide improved treatment outcomes in diabetic rats compared to when antidiabetics were used alone.

Key words: Diabetes mellitus, Antidiabetics, Antihypertensives 